\title{
Energy dissipation through entrained air compression in plunging jets
}

\begin{abstract}
The energy dissipation in plunge pools is often related to air entrainment in the plunging jet. Recent experimental work showed that the void fractions below the impinging jet are significantly smaller than outside the jet impact. This was attributed to the compression of the entrained air bubbles. In this paper, the thermodynamics of air bubble compression is introduced as a novel energy dissipation mechanism in civil engineering hydraulics. For the analysis of reported experimental data, isothermal conditions were applied as a lower bound approximation. It was found, that the energy dissipation rates range from 2.7 to $9.7 \%$ for a jet velocity of $10 \mathrm{~m} \mathrm{~s}^{-2}$ and void fractions of 0.11 and 0.31 , to 15.9 to $36.0 \%$ for a jet velocity of $24.5 \mathrm{~m} \mathrm{~s}^{-1}$ and void fractions of 0.35 to 0.55 . The results may explain the difference between energy dissipation rates in plunge pools at model and full scale.
\end{abstract}

Keywords: Aerated flow; bubble dynamics; energy dissipation; multiphase flow; similarity (scaling) theory

\section{Introduction}

Plunge pools serve as energy dissipating elements for dams and reservoirs. In these pools, the energy of an impinging water jet is dissipated through turbulence. The hydraulics of plunge pools and in particular the pressures generated by the impinging jet have been researched thoroughly, e.g. by Ervine, Falvey \& Withers (1997).

The energy dissipation in plunge pools appears to be significantly larger at full scale than at model scale. This is attributed to the jet aeration, which is significantly higher at full scale velocities than at model scale, see e.g. Bretschneider (1965), Kobus (1980), Pfister and Chanson (2014). An actual aeration related energy dissipation mechanism has however as yet not been described. This article aims to introduce a hitherto neglected energy dissipation mechanism in plunge pools, namely the isothermal compression and subsequent expansion of air bubbles during the jet entry and diffusion in the plunge pool

The thermodynamics of compressed air bubbles will be employed as a novel concept for the analysis of energy dissipation in aerated flow in civil engineering hydraulics. Initially, the work required for compression and released during the expansion of air bubbles will be considered. Reasons for assuming isothermal conditions as a lower bound assumption and the specifics of the thermodynamics of plunge pools are given. The picture will then be put together by analysing experimental results reported by Bollaert, Manso and Schleiss (2009). Their analysis of the tests will be expanded, and the results interpreted in the context of scale effects in plunge pool model tests. 


\section{The compression of air bubbles}

During the impact of a plunging jet onto a water cushion, air is entrained and pressures are generated. The pressure increase means that any air entrained in the jet will be compressed.

\subsection{Volume change}

A change in pressure changes the volume of an air bubble surrounded by water. The volume change also can lead to an increase in temperature. In the experiments reported by Bollaert et al. (2009) however, neither bubble diameters nor temperatures were recorded, so that a more detailed analysis is difficult.

There are however two limiting and a transitional condition for the compression of air bubbles, namely isothermal and adiabatic compression:

\subsection{Condition 1: Isothermal compression, lower limit}

During the compression, all heat is transferred immediately into the environment and the temperature of the air remains constant at ambient temperature. Similarly, during expansion heat is drawn in from the environment, and again the temperature of the bubble remains at the ambient value. The volume change from an initial volume $V_{1}$ to the end volume $V_{2}$ for a change in pressure from $p_{1}$ to $p_{2}$ is given by the ideal gas law as

$$
V_{2}=V_{1} \frac{p_{1}}{p_{2}}
$$

The external work $W_{\text {iso }}$ required for the isothermal compression can then be determined as:

$$
W_{\text {iso }}=p_{1} V_{1} \ln \left(\frac{p_{1}}{p_{2}}\right)-p_{1}\left(V_{1}-V_{2}\right)
$$

Where $V_{1}$ is the initial volume, and $V_{2}$ the volume after compression. The pressures $p_{1}$ and $p_{2}$ are hereby in absolute values. The r.h.s. second term in Eq. (2) constitutes the atmospheric work, which has to be subtracted, since all processes are assumed to begin and end at ambient pressure.

\subsection{Condition 2: adiabatic compression, upper limit}

During the compression, no heat exchange takes place and the air bubble heats up. During expansion, the temperature returns to ambient. The relationship of pressure and volume is given in Eq. (2) where the isentropic coefficient $\kappa$ is taken as $\kappa=1.4$ for air:

$p_{1} V_{1}^{\kappa}=p_{2} V_{2}^{\kappa}$ 
For a given compression ratio $V_{1} / V_{2}$ the equation can be written as:

$$
p_{2}=\left(\frac{V_{1}}{V_{2}}\right)^{\kappa} p_{1}
$$

With this equation, the pressure required for a given compression ratio can be determined.

The adiabatic work $W_{a d}$ can then be calculated as follows:

$$
W_{a d}=\frac{1}{1-\kappa}\left(p_{1} V_{1}-p_{2} V_{2}\right)-p_{1}\left(V_{1}-V_{2}\right)
$$

\subsection{Condition 3: polytropic compression, transitional}

In many technical applications, some heat exchange takes place during compression or expansion. In our case, heat and work flows move in opposite directions. This is usually modelled by choosing the isentropic coefficient to have a value in between $>1$ (i.e. near isothermal conditions), and 1.4 (adiabatic conditions), see e.g. Dietzel and Wagner (2004).

\subsection{Analysis}

Figure 1 shows the work required for the compression of an initial air volume $V_{1}=1 \mathrm{~m}^{3}$ for isothermal conditions and for different isentropic coefficients from $\kappa=1.1$ to $\kappa=1.4$. The compression coefficient $n$ is defined as $n=V_{1} / V_{2}$, i.e. the ratio of uncompressed and compressed air volume. The work per $\mathrm{m}^{3}$ was calculated using Eqs (2) and (5).

It can be seen, that the work required increases with increasing value of the coefficient, and that the isothermal compression requires the least work. For the following analysis, isothermal conditions will therefore be employed as a conservative or lower bound assumption for the work required for the compression of the air bubbles in water. Here it needs to be added that the rate of heat transfer depends on the ratio of thermal conductivities of the fluids involved, for water and air this ratio is 23 . The heat capacity of water is 3,011 times larger than that of air. The compression of an initial water-air volume of $1 \mathrm{~m}^{3}$ with an air content of $0.55 \mathrm{~m}^{3}$ (i.e. an aeration ratio of 0.55 ), with a compression ratio of $1: 5$ leads to a temperature increase of the water of only by $0.068 \mathrm{~K}$. This assumes a complete transfer of the heat generated during the compression of the air.

\section{The compression of air bubbles}

Very little work has been reported on the thermodynamics of aerated flow in civil engineering applications, although some of the potential effects are interesting. These include the energy dissipation in plunge pools and stepped spillways caused by compression and expansion of air 
bubbles, or the possibility of supersonic flow in spillways. The thermodynamics of aerated flow situations in civil engineering are characterised by the following properties:

(1) They are usually gravity driven, and the energy flow is unidirectional from higher to lower energy levels.

(2) The system is an open thermodynamic system where mass and energy flows enter and exit. Air compression takes place in an enclosed space, whilst expansion can occur in an open environment, often with a free surface.

Figure 2a shows a typical system. Aerated water flows through an opening ' $O$ ' into a shallow reservoir. Because of the pressure applied by the piston $P$ (which is larger than the hydrostatic pressure generated by the water depth $d$ at the outflow), and the flow resistance at the opening, the air bubbles in the pipe are compressed. Additional compression work $F \times \Delta x$ is required to drive the flow. As soon as the bubbles enter the free reservoir ' $R$ ', the open side of the system, the bubbles expand in all directions, and rise to the surface due to their buoyancy. In Fig. 2b, a model for the expansion of an air bubble is shown. The expansion takes place as the bubble moves into a zone of lower pressure following the main flow direction. The expansion generates a displacement of the surrounding fluid. However, only the displacement in the direction of the flow field will return energy to the flow. Since the bubble is assumed to expand evenly in all directions, i.e. $x$ (main direction of flow), $y$ and $z$, only one third of the compression energy is returned to the main flow, the other two thirds are diffused in a 360 degree and expanding circle normal to the direction of the flow and into the reservoir.

\section{Experiments}

So far, the principles of an energy dissipation mechanism connected with the compression of air bubbles in water have been discussed. The next question is, whether or not this mechanism can make significant contributions to the overall energy dissipation in plunge pools. Bollaert et al. (2009) reported experiments on a plunging jet impinging onto a reservoir of $0.67 \mathrm{~m}$ depth with velocities of 6.1 to $24.5 \mathrm{~m} \mathrm{~s}^{-1}$. The ratio of water depth $Y$ to jet diameter $D$ for the measurements given was $Y / D=9.3$ (Fig. 3a).

\subsection{Void fraction}

Void fractions were measured at three locations, within the free flow at point ' 1 ', point '2' at the stagnation point and at point ' 3 ' in the impinging jet (Fig. 3a).

In the free stream at point ' 1 ', the void fraction increased with increasing jet velocity from 0.05 for $v_{0}=6 \mathrm{~m} \mathrm{~s}^{-1}$ to 0.35 for $v=20$ to $24 \mathrm{~m} \mathrm{~s}^{-1}$ (Fig. 3b). With increasing jet velocity, 
the void fraction at measurement point ' 2 ' increased rapidly from 0.02 to 0.08 , and then remained practically constant and independent of the jet velocity. At point ' 3 ', the void fractions were slightly higher with values of 0.04 to 0.12 . This was attributed to the compression of the air entrained during jet entry into the water cushion, and the subsequent expansion when the air bubbles reached zones outside the effect of the impinging jet. Using the ideal gas law, Bollaert et al. (2009) demonstrated that this is indeed plausible, although they only considered the compression ratio between points ' 3 ' (jet) and ' 1 ' (free stream). Their results indicated a pressure of $0.5 v_{0}^{2} / 2 g$ at ' 3 '.

However, a comparison between the stagnation point ' 2 ' and the free stream at ' 1 ' appears to be more interesting. Following Bollaert et al. (2009), and keeping the assumption of isothermal compression as a lower bound estimate as discussed in section 2 in mind, the compressed void fraction $V_{2}$ at point ' 2 ' was determined from the void fraction at atmospheric pressure $V_{1}$ at point ' 1 ' using Eq. (2). The pressure $p_{2}$ at point ' 2 ' was calculated as a function of the velocity head of the impinging jet and a pressure coefficient $C_{P}=1.3$ for a ratio of water depth $Y$ and jet thickness $D$ of $Y / D=9.3$, see e.g. Ervine et al. (1997):

$p_{2}=C_{P} \rho_{w} \frac{v_{0}^{2}}{2 g}+0.67 \rho_{w} g$

where $\rho_{w}$ is the density of water. The pressure coefficient indicates that it is not only the mean pressure which affects the compression of the air bubbles, but also the dynamic component. Figure $3 b$ shows the measured void ratios at points ' 1 ' and ' 2 ' as a function of the jet velocity. The theoretical values for the compressed void fractions at point ' 2 ', calculated as described above, are also shown as open squares. They agree nicely with the measured values. For comparison, Fig. $3 \mathrm{c}$ shows the situation for adiabatic compression. In order to map the void fractions at ' 1 ' onto ' 2 ', a pressure coefficient of $C_{P}=3.0$ is required.

Here, we can return to the original assumption of isothermal compression of air as a lower bound approach. The pressure coefficient $C_{P}$ required for isothermal compression was $C_{P}=1.3$, which is within the range for such coefficients given in the literature. Assuming adiabatic conditions, a coefficient of $C_{P}=3.0$ would be needed to achieve the compression ratios reported by Bollaert et al. (2009). This is far higher than the pressure coefficients recommended in the literature and again supports the assumption of an isothermal process. The tests conducted by Bollaert et al. (2009) also showed that the aeration is a function of scale, increasing with increasing jet velocity or scale. 


\subsection{Compression work}

The work required for the compression of the entrained air fraction will now be determined for the pressures and void fractions from the experiment, Fig. 3b, using Eq. (6) to determine the pressure and Eqs (2) and (5) to calculate the compression work. For every jet velocity $v$, there are two void fractions: $\alpha_{1}$ (uncompressed) and $\alpha_{2}$ (compressed), determined from Fig. $3 \mathrm{~b}$. With these void fractions, and with $p_{1}=100 \mathrm{kN} \mathrm{m}^{-2}$, the pressure $p_{2}$ can be determined:

$p_{2}=p_{1} \frac{\alpha_{1}}{\alpha_{2}}$

With $V_{1}=1 \mathrm{~m}^{3}$ initial uncompressed air, using Eq. (3), and assuming that $1 / 3$ of the expansion work is returned to the flow (see Fig. 2b), the work $W_{\alpha}$ required for compression of an initial void fraction $\alpha_{1}$ becomes

$$
W_{\alpha}=\frac{2}{3} \alpha_{1} W_{c o m p}
$$

The potential energy $E_{p o t}$ for $1 \mathrm{~m}^{3}$ of water-air mixture is then determined assuming that the potential energy of the air fraction is negligible. With a height $H=v_{0}^{2} / 2 g$, the potential energy is

$$
E_{p o t}=(1-\alpha) \rho_{w} g H
$$

The energy dissipation ration $n$ is the ratio of compression work required for the air fraction, and total potential energy of the flow:

$$
n=\frac{W_{\alpha}}{W_{p o t}}
$$

The dissipation ratio indicates how much of the total energy of the flow is dissipated in air compression and subsequent expansion, i.e. how much of the energy is removed from the flow. It is shown in Fig. 4 for the isothermal (Fig. 4a), and the adiabatic case (Fig. 4b). The pressures below the impinging jet as determined from the literature suggest that the actual condition is very close to isothermal, see section 2.3. In the following therefore only this condition will be considered.

The maximum void ratio in the experiment was 0.36 for a jet velocity of $v_{0}=22.2 \mathrm{~m}$ $\mathrm{s}^{-2}$. Air entrainment only took place at the point where the jet hit the reservoir surface, since the jet issued from a pipe around $0.7 \mathrm{~m}$ above the reservoir. In a real situation, a water jet from an overflow will fall freely for tens of meters, breaking up and entraining more air along its falling length. To simulate this effect, the maximum void fraction was therefore only increased by 0.1 and 0.2 to 0.45 and 0.55 . A part of the jet core will however remain compact, 
as can be seen from the erosion of the pool bottom e.g. at the Kariba dam, Bollaert et al. (2012). The energy dissipation caused by air compression appears to have no effect on the bed erosion process. Figure 4a shows the relevant energy dissipation ratios as well for isothermal conditions. It can be seen that:

(1) For flow velocities below $10 \mathrm{~m} \mathrm{~s}^{-2}$, and corresponding low aeration ratios, the energy dissipation of the air fraction is small to negligible. The energy dissipation ratio reaches 2.7 to $9.7 \%$ for $v_{0}=10 \mathrm{~m} \mathrm{~s}^{-2}$.

(2) For higher, near full scale velocities of $v_{0}=24 \mathrm{~m} \mathrm{~s}^{-2}$, the dissipation ratio increases and reaches 15.9 to $36.0 \%$.

As an answer to the initial question it can be stated that at full scale the energy dissipation through compression of entrained air can contribute significantly to the overall energy dissipation, whilst at small / model scale the effect will be small.

\subsection{Scaling}

The purpose of this paper is to introduce a thermodynamic energy dissipation mechanism in aerated flow. The experiments indicated that aeration increases with increasing jet velocities, and the analysis showed that energy dissipation increases exponentially with increasing velocities. This leads to the conclusions that air compression related energy dissipation will be relatively higher at full than at model scale. There are however two more aspects which should briefly be assessed to ensure that there are no additional scale effects contained in the experiment and its interpretation. In particular, minimum values of Reynolds number Re and the Weber number We are required (Pfister and Chanson, 2014). The Weber number is given as

$\mathrm{We}=\frac{\rho v_{0}^{2} h}{\sigma}$

where $\rho$ is the density of water, $v_{0}$ the velocity of the air water flow, $h$ the flow depth of the water-air mixture and $\sigma$ the surface tension of water $\left(72.8 \mathrm{mN} \mathrm{m}^{-1}\right)$. For similarity, either of the condition $\mathrm{We}^{0.5}>110$ to 170 or $\mathrm{Re}>1.5 \times 10^{5}$ to $3.0 \times 10^{5}$ need to be fulfilled. In line with Pfister and Chanson (2014), the Reynolds and the Weber numbers were calculated using the water depth $Y=0.67 \mathrm{~m}$ as characteristic length $h$. In the experiment reported by Bollaert et al. (2009), $\mathrm{We}^{0.5}$ ranges from 305 to 1127 . The Reynolds number was determined with:

$\operatorname{Re}=\frac{\rho_{w} v_{0} Y}{\mu}$ 
where $\mu$ is the dynamic viscosity of water of $1.0 \times 10^{-3} \mathrm{~Pa} \cdot \mathrm{s}$. The Reynolds number ranges from $3.4 \times 10^{6}$ for $v_{0}=5 \mathrm{~m} \mathrm{~s}^{-1}$ to $1.64 \times 10^{7}$ for $v_{0}=24.5 \mathrm{~m} \mathrm{~s}^{-1}$. It can therefore be assumed that air entrainment itself was scaled.

Heller (2017) discussed the topics of self similarity and Reynolds invariance in Froude scaled models. "A phenomenon is called self-similar if the spatial distribution of its flow properties at various different instances in time (and / or spatial locations) can be obtained from one another by a similarity transformation.", see Heller (2017). Re-invariance is given if the effect of viscosity is negligible. These are interesting concepts, and the fact that the Reynolds number of the experiments by Bollaert et al. (2009) is (a) above the limits given in Pfister and Chanson (2014) and (b) one order of magnitude below the full scale values may indicate that air entrainment and possibly the dissipation of the energy radiated by the expanding air bubbles are modelled accurately. Finally, it should be mentioned that viscous energy dissipation increases with increasing bubble concentration, e.g. Das (2012). This may increase the energy dissipation of the energy radiated by the expanding bubbles at full scale further.

\section{Discussion}

\subsection{Experiments}

The experiments reported by Bollaert et al. (2009) convincingly demonstrated that significant air bubble compression can occur during the entry of a plunging jet into a water cushion. They also quantified the void fraction and found that it increases with increasing jet velocity. A further look at the thermodynamic characteristics of such a situation showed, that a plunge pool constitutes an open thermodynamic system, where different boundary conditions for compression and expansion exist. This leads to the partial dissipation of the compression work in the expansion zone.

The aeration ratio increases near linearly with the jet velocity. The non-linear compressibility of air means, that the relative energy dissipation increases exponentially with increasing pressure and jet velocity.

For physical model tests of aerated flows this means that energy dissipation ratios will be substantially lower at model than at full scale. The isothermal compression of the air fraction, assumed as a lower bound estimate for the energy dissipation, can therefore at least partially explain the scale effects described in the literature (Bretschneider 1965, Heller 2011, Pfister and Chanson 2014). The analysis indicates that an artificial increase of air volume at model scale will not achieve near full scale conditions. 


\subsection{System boundaries}

One boundary of the system is the plunge pool floor. In real plunge pools, this floor often consists of rock which contains fissures, which are filled with an air - water mixture. The dynamic pressures generated by the plunging jet impact can travel into the fissures as compression waves, and erode the rock from within, e.g. Duarte et al. (2015). In certain conditions, the fluctuations of the pressure can even induce a dynamic resonance inside the fissures, leading to increased erosion.

In the experiments reported by Duarte et al. (2015) the ratio of fissure area and bottom area was approximately 0.01 . Taking this value as typical, the area of fissures can be considered as small compared with the overall floor area. The lower boundary therefore becomes rigid. This implies that the influence of fissures on the pressure generation and air compression is small. Decompression of the air bubbles are only occurs during the transition of the air bubbles into the reservoir, so that the effect of decompression on pressures in cracks or fissures can be expected to be small.

\subsection{Further work}

The investigation of thermodynamic energy dissipation in Civil Engineering Hydraulics will require fundamental experiments in order to assess the validity of theoretical results, and to provide actual quantitative information. The practical problem for future experiments here is the necessity for near full scale velocities and aeration ratios, which makes the construction of specialised facilities as e.g. employed by Duarte et al. (2015) necessary. In addition, the measurement of void fraction and, if possible, bubble diameters and temperatures as well as the determination of energy losses is required. The first stage could be a variation of the tests described by Bollaert et al. (2009) whereby the jet is introduced slightly below the reservoir water surface as benchmark experiment (no or very little aeration).

Flow velocities at selected points in the reservoir and surface distortions (waves) are measured as indicators of the energy transfer from jet to reservoir as is implicitly suggested in Pfister and Chanson (2014) (Fig. 5). Lifting the pipe exit above the reservoir will generate an aeration ratio similar to Bollaert et al. (2009). Further aeration could be added by inserting a Venturi section with an opening into the pipe. This will draw air into the water jet. By increasing the aeration ratio whilst maintaining energy input, energy dissipation ratios could be quantified. Hereby, the change in momentum created by the reduced density needs to be taken into account, see Duarte et al. (2016). Velocity and turbulence measurements would allow to get more information about the flow situation. 
The scale effects can probably only be addressed with numerical models whereby the water - air mixture is modelled as a compressible two-phase medium. This would however probably only be meaningful once more detailed experimental information is available.

\section{Conclusions}

The difference in energy dissipation between model and full scale in plunge pools is usually attributed to the aeration content, which is lower at model scale, without a dissipation mechanism having been described so far. In this article, the thermodynamics of compression and expansion of air bubbles was introduced as a novel concept in the analysis of energy dissipation in aerated flow. From the theoretical considerations outlined in the manuscript, the following conclusions can be drawn:

- In plunge pools, air bubbles are compressed by the pressure generated by the impact of the impinging jet. Isothermal conditions provide a lower bound estimate of the compression work required.

- During the expansion of the bubbles, a substantial amount of the compression work is dissipated.

- The energy dissipation rate, i.e. the ratio of compression work and available potential energy of the flow increases with increasing aeration content and void fraction. It reaches 2.6 to $9.1 \%$ for a jet velocity of $10 \mathrm{~m} \mathrm{~s}^{-1}$ and initial void fractions of 0.12 and 0.32 , and 13.4 to $30.1 \%$ for a jet velocity of $24.5 \mathrm{~m} \mathrm{~s}^{-1}$ and void fractions of 0.36 to 0.55 .

- The energy dissipation rate increases exponentially with the absolute pressure magnitude. This may have significant consequences for the interpretation of model tests results.

- Experimental verification of the theoretical analysis is required. 


\section{Notation}

$C_{P}=$ pressure coefficient (-)

$D=$ jet thickness (m)

$E=\operatorname{energy}(\mathrm{J})$

$F=$ force $(\mathrm{N})$

$g=$ gravity acceleration $\left(\mathrm{m} \mathrm{s}^{-2}\right)$

$n=$ energy dissipation ratio $(-)$

$P=$ pressure $(\mathrm{Pa})$

$\mathrm{Re}=$ Reynolds number $(-)$

$T=$ temperature $(\mathrm{K})$

$v=$ velocity $\left(\mathrm{m} \mathrm{s}^{-1}\right)$

$V=\operatorname{air}$ volume $\left(\mathrm{m}^{3}\right)$

$W=\operatorname{work}(\mathrm{J})$

We $=$ Weber number $(-)$

$Y=$ water depth $(\mathrm{m})$

$\alpha=$ aeration ratio $(-)$

$\kappa=$ isentropic coefficient (-)

$\mu=$ dynamic viscosity of water $(\mathrm{Pa} \mathrm{S})$

$\rho_{w}=$ density of water $\left(\mathrm{kg} \mathrm{m}^{-3}\right)$

$\sigma=$ surface tension of water $\left(\mathrm{mN} \mathrm{m}^{-1}\right)$ 


\section{References}

Bollaert, E. F., Manso, P. A., \& Schleiss, A. J. (2009). Discussion of "Effect of jet aeration on hydrodynamic forces on plunge pool floors". Appeared in the Canadian Journal of Civil Engineering 35 (5), 521-530. Canadian Journal of Civil Engineering, 36(3), 524-526.

Bollaert, E., Duarte, R., Pfister, M., Schleiss, A. and Mazvidza, D., 2012. Physical and numerical model study investigating plunge pool scour at Kariba Dam. Proceedings of the 24th Congress of CIGB-ICOL. Paper Q. 94 - R. 17. Kyoto.

Bretschneider H. (1965). Die Auswirkungen von Oberflächenkräften im wasserbaulichen Versuchswesen (The effects of surface tension forces in hydraulic modelling, in German). Bautechnik, 42(4), 126-130.

Das, D. (2012). Study of air bubble injection into laminar and turbulent water flow through an annular section. Master's Thesis. City University of New York.

Dietzel, F., \& Wagner, W. (2013). Technische Wärmelehre. Leipzig: Vogel Buchverlag.

Ervine, D. A., Falvey, H. T., and Withers, W. (1997). Pressure fluctuations on plunge pool floors. Journal of Hydraulic Research, 35(2), 257-279.

Duarte, R., Schleiss, A.J. and Pinheiro, A., 2015. Influence of jet aeration on pressures around a block embedded in a plunge pool bottom. Environmental Fluid Mechanics, 15(3), 673-693.

Duarte, R., Pinheiro, A., \& Schleiss, A. J. (2016). Dynamic response of an embedded block impacted by aerated high-velocity jets. Journal of Hydraulic Research, 54(4), 399-409.

Heller V. (2011). Scale effects in physical hydraulic engineering models. Journal of Hydraulic Research, 49(3), 293-306.

Heller, V. (2016) Self-similarity and Reynolds number invariance in Froude modelling. Journal of Hydraulic Research, 55 (1). 1-17.

Kobus H. (Ed.) (1980). Hydraulic Modelling. Hamburg: Parey Verlag.

Pfister, M., \& Chanson, H. (2014). Two-phase air-water flows: Scale effects in physical modelling. Journal of Hydrodynamics, Ser. B, 26(2), 291-298. 


\section{List of figures}

Figure 1 Compression work required for $1 \mathrm{~m}^{3}$ of air as function of compression ratio and thermal exchange

Figure 2 Thermodynamic systems in civil engineering hydraulics: (a) Inflow into reservoir, (b) Bubble expansion and fluid displacement

Figure 3 Void fraction measurements (Bollaert et al., 2009): (a) Experimental set-up, (b) Void fractions at points ' 1 ' and '2' - isothermal, (c) Void fractions at points ' 1 ' and ' 2 ', adiabatic

Figure $4 \quad$ Energy dissipation ratio for isothermal and adiabatic conditions: (a) Isothermal compression, (b) Adiabatic compression 


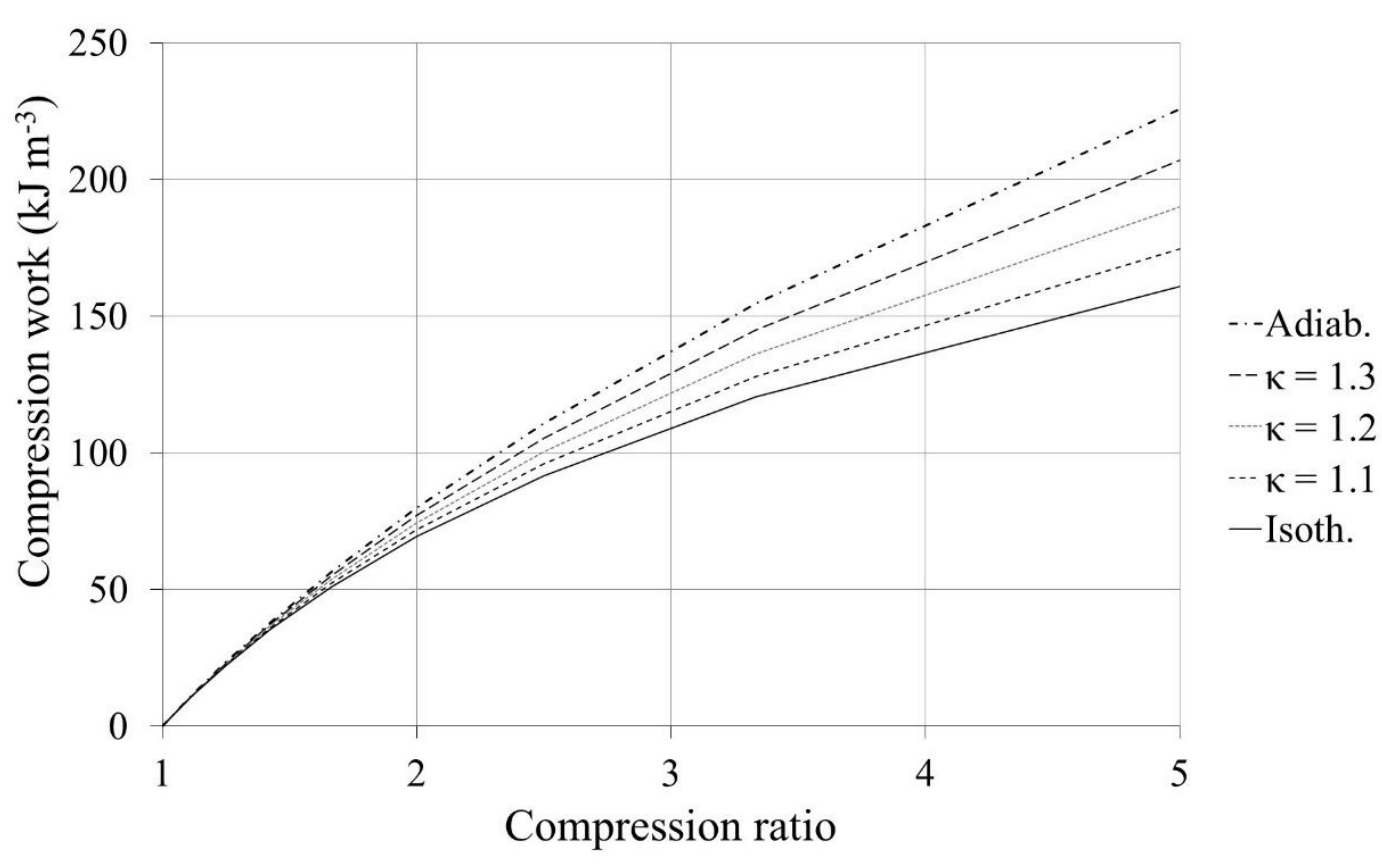

Figure 1 Compression work required for $1 \mathrm{~m}^{3}$ of air as function of compression ratio and thermal exchange 
(a)

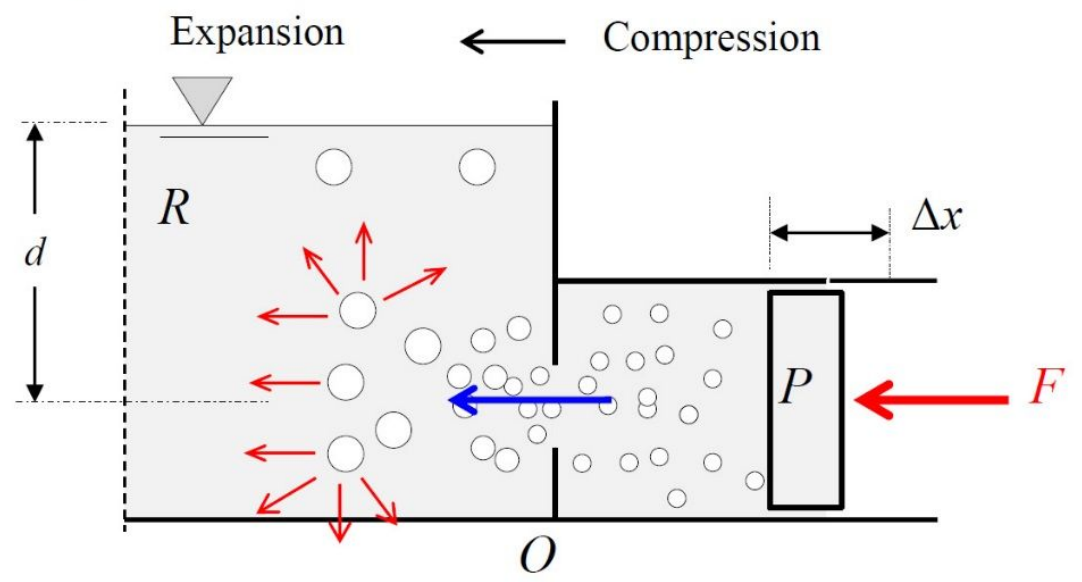

(b)

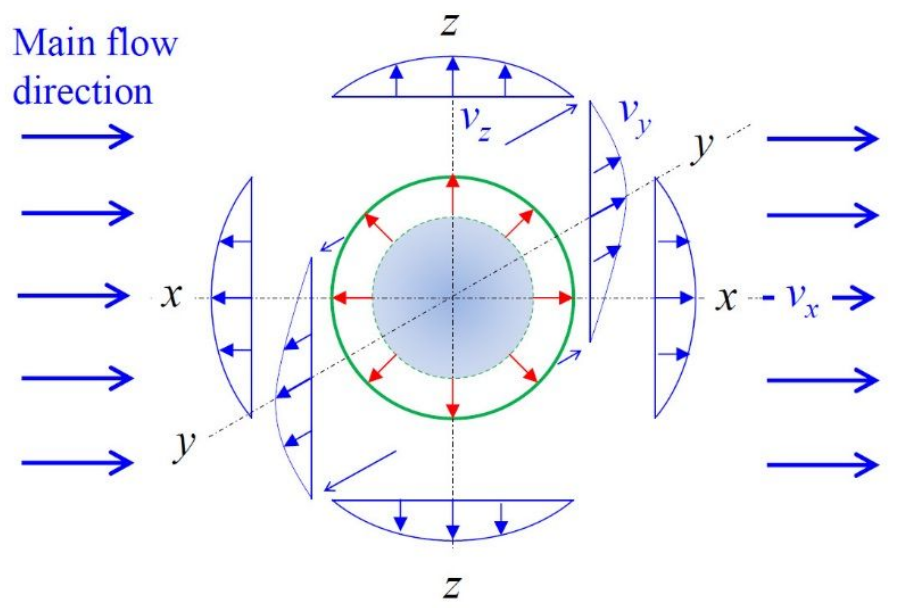

Figure 2 Thermodynamic systems in civil engineering hydraulics: (a) Inflow into reservoir, (b) Bubble expansion and fluid displacement 


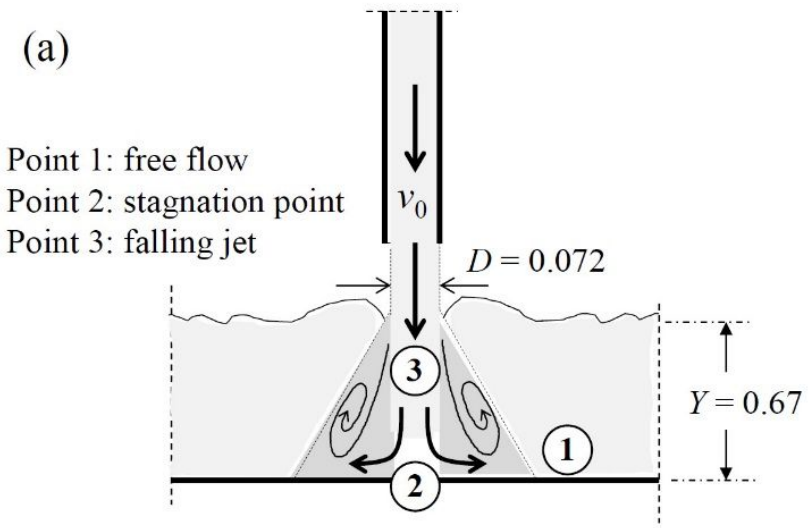

(b)
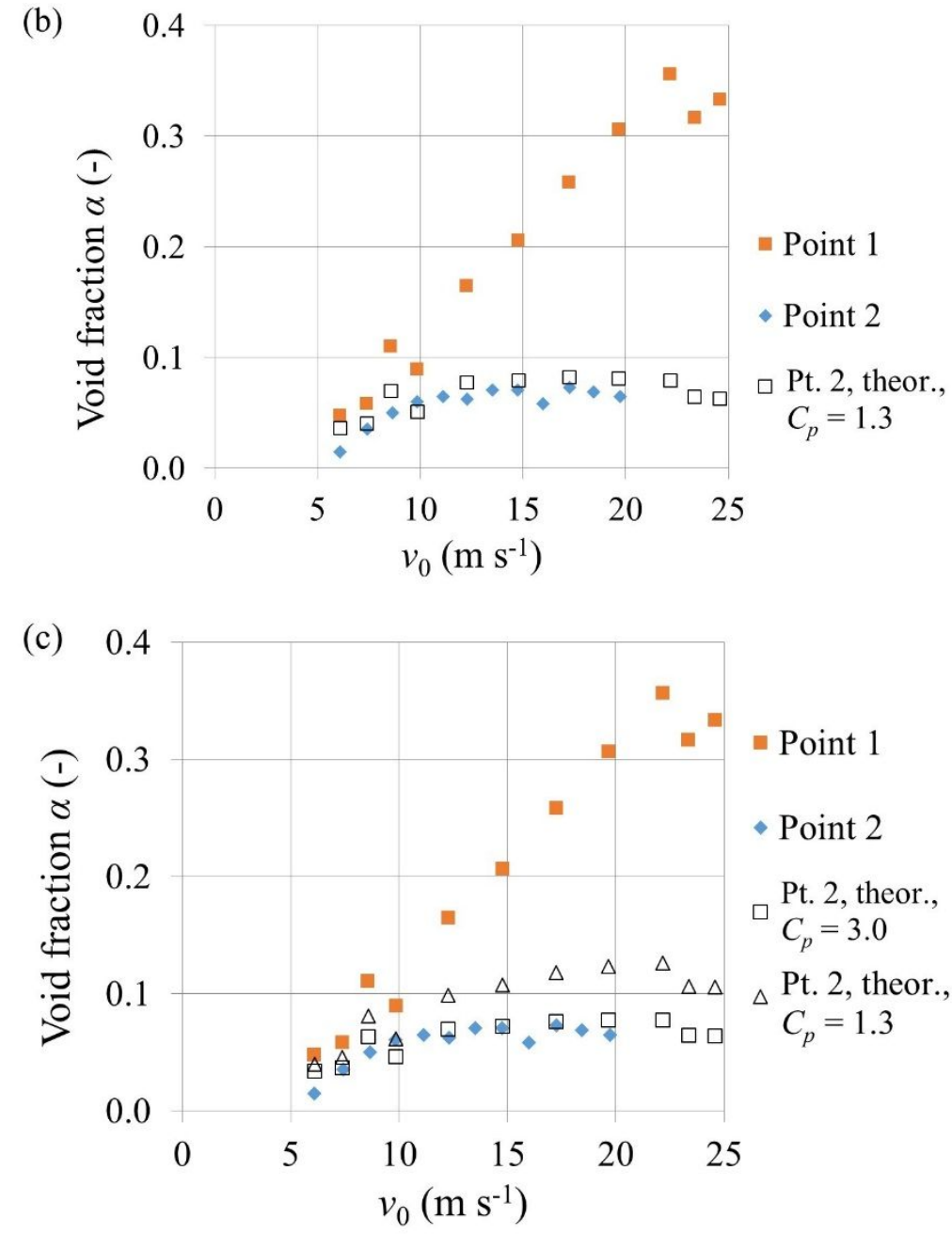

Figure 3 Void fraction measurements (Bollaert et al., 2009): (a) Experimental set-up, (b) Void fractions at points ' 1 ' and '2' - isothermal, (c) Void fractions at points ' 1 ' and ' 2 ', adiabatic 

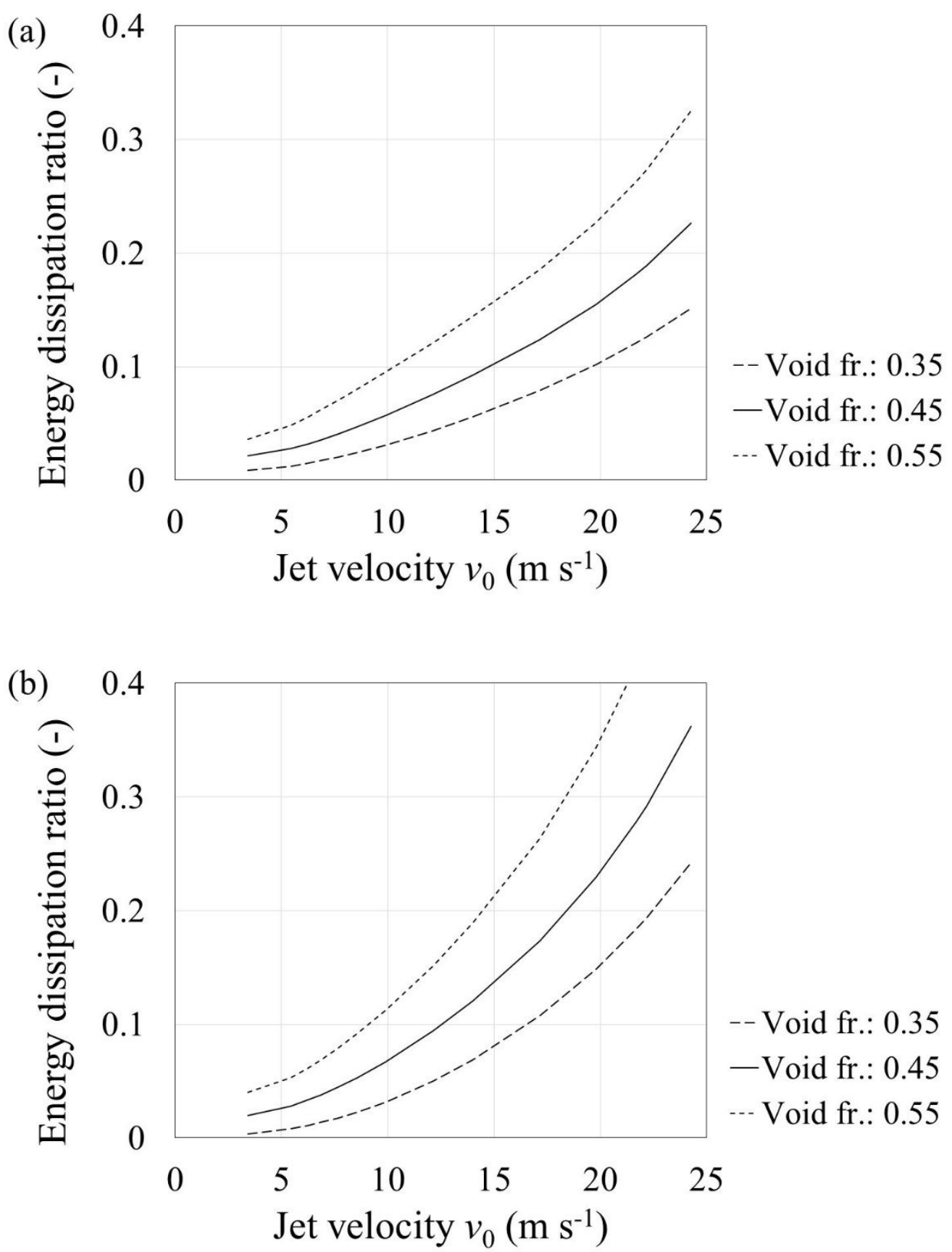

Figure 4 Energy dissipation ratio for isothermal and adiabatic conditions: (a) Isothermal compression, (b) Adiabatic compression 


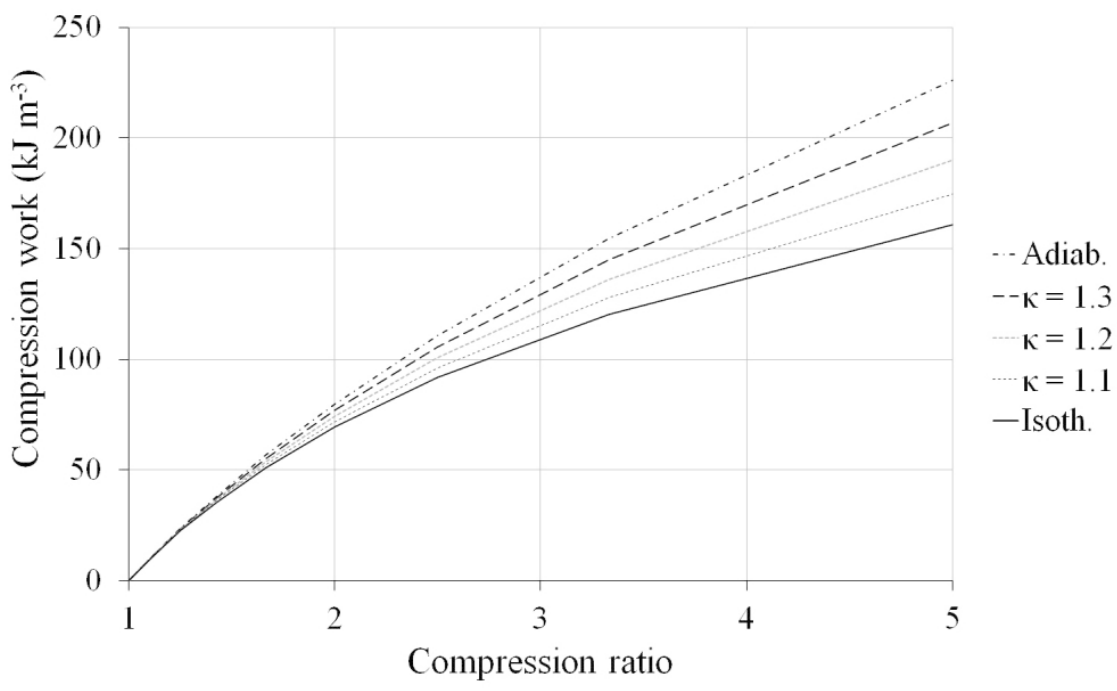

Figure 1 Compression work required for $1 \mathrm{~m} 3$ of air as function of compression ratio and thermal exchange $145 \times 86 \mathrm{~mm}(300 \times 300$ DPI $)$ 
(a)

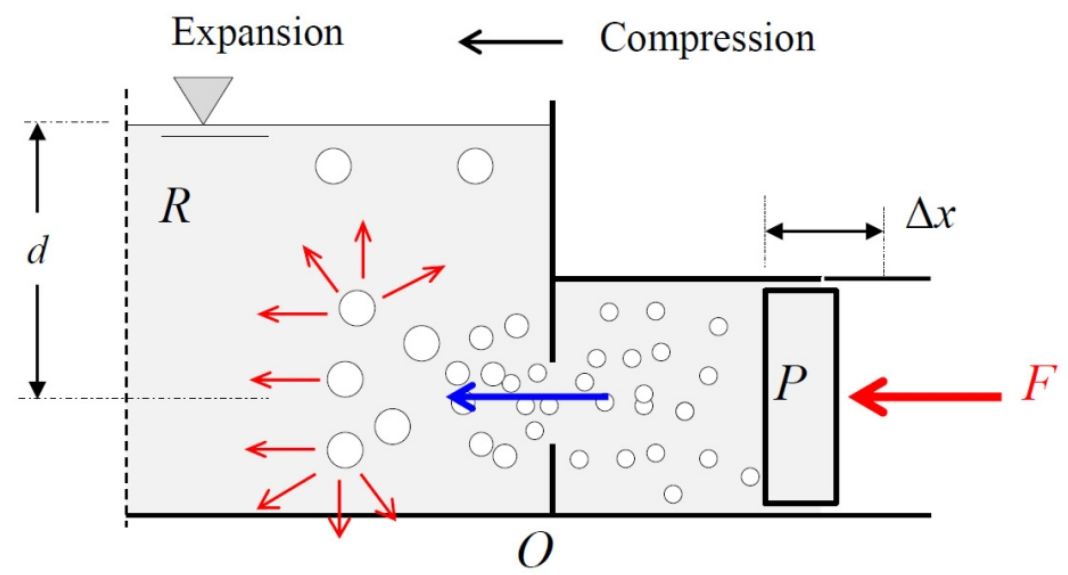

Figure 2 Thermodynamic systems in civil engineering hydraulics: (a) Inflow into reservoir $115 \times 70 \mathrm{~mm}(300 \times 300 \mathrm{DPI})$ 
(b)

Figure 2 Thermodynamic systems in civil engineering hydraulics: (b) Bubble expansion and fluid displacement $114 \times 80 \mathrm{~mm}(300 \times 300$ DPI $)$

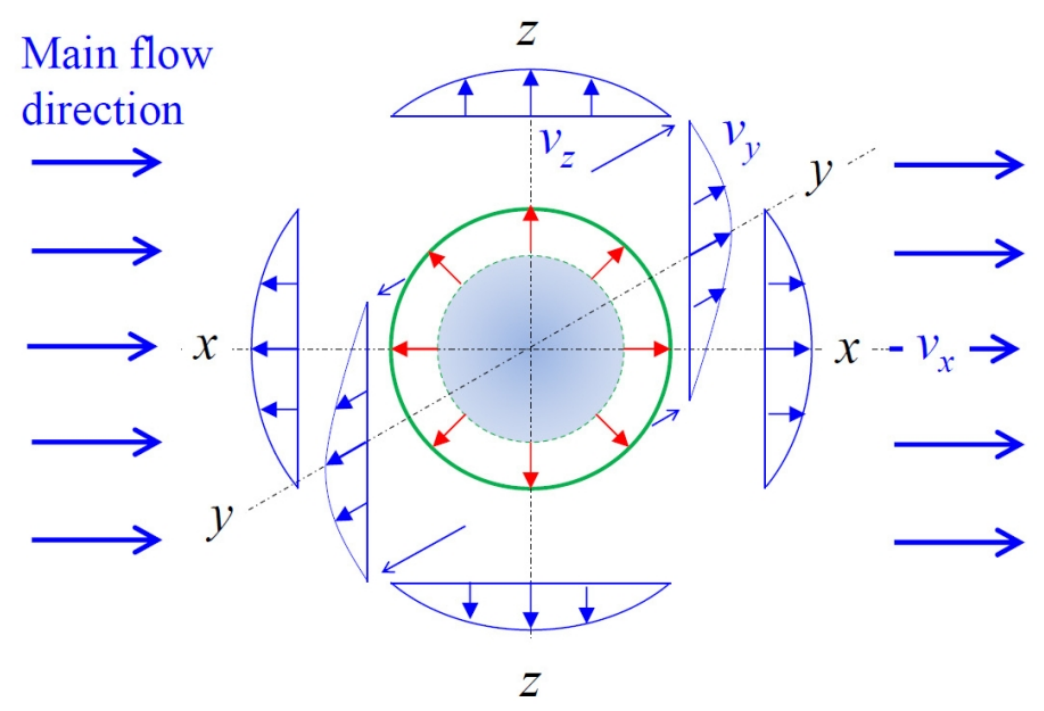




\section{(a)}

Point 1: free flow Point 2: stagnation point Point 3: falling jet

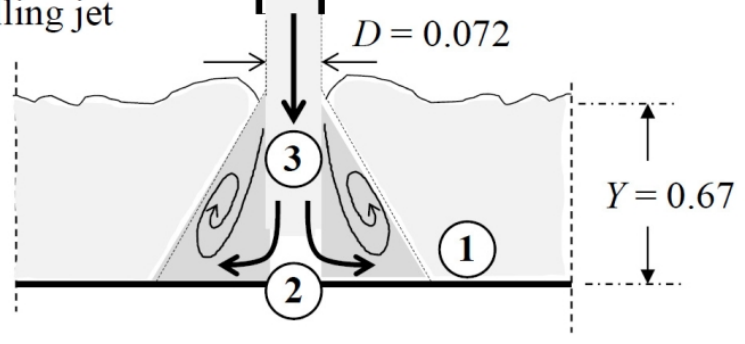

Figure 3 Void fraction measurements (Bollaert et al., 2009): (a) Experimental set-up $137 \times 88 \mathrm{~mm}(300 \times 300$ DPI $)$ 
(b)

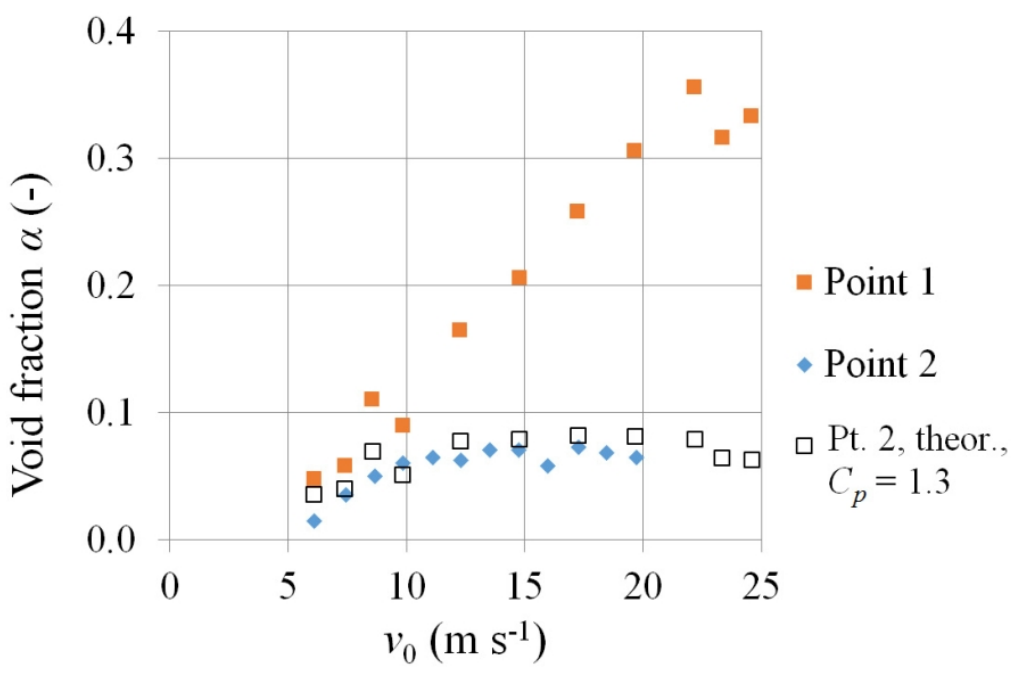

Figure 3 Void fraction measurements (Bollaert et al., 2009): (b) Void fractions at points ' 1 ' and ' 2 ' isothermal $118 \times 76 \mathrm{~mm}(300 \times 300 \mathrm{DPI})$ 


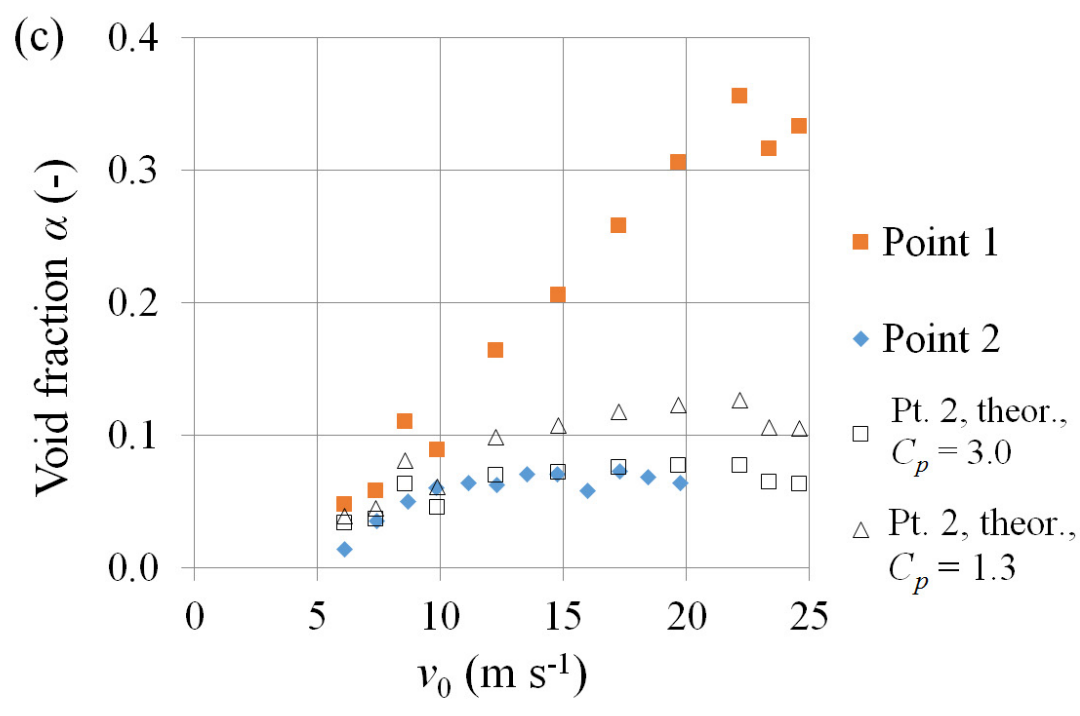

Figure 3 Void fraction measurements (Bollaert et al., 2009): (c) Void fractions at points '1' and '2', adiabatic $113 \times 73 \mathrm{~mm}(300 \times 300 \mathrm{DPI})$ 


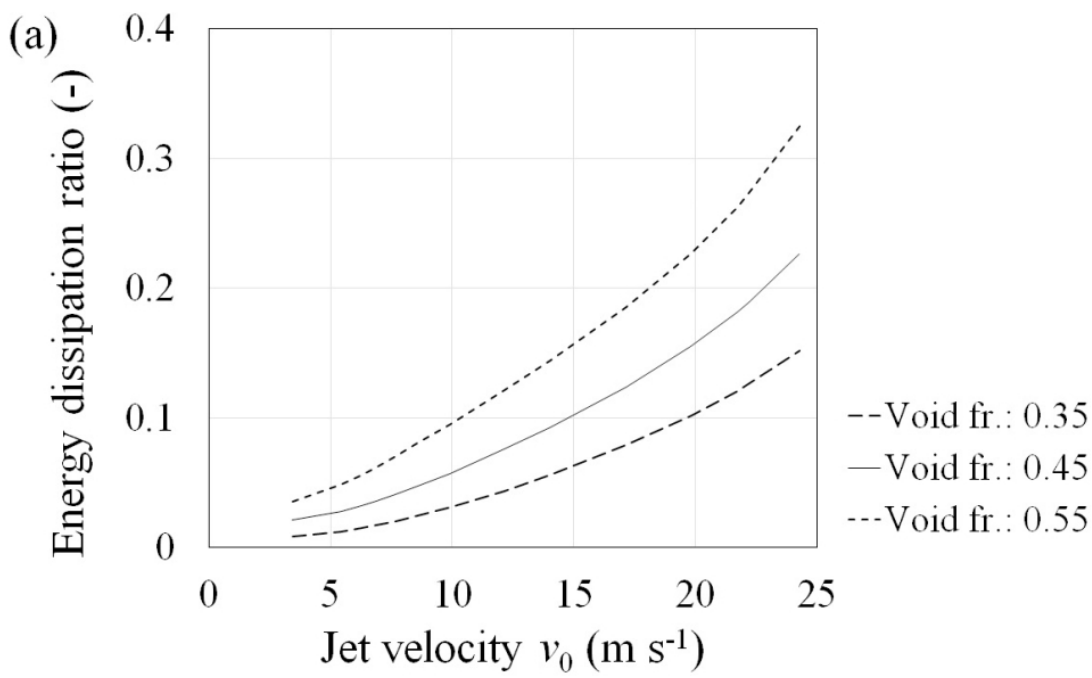

Figure 4 Energy dissipation ratio for isothermal and adiabatic conditions: (a) Isothermal compression $116 \times 77 \mathrm{~mm}(300 \times 300$ DPI) 


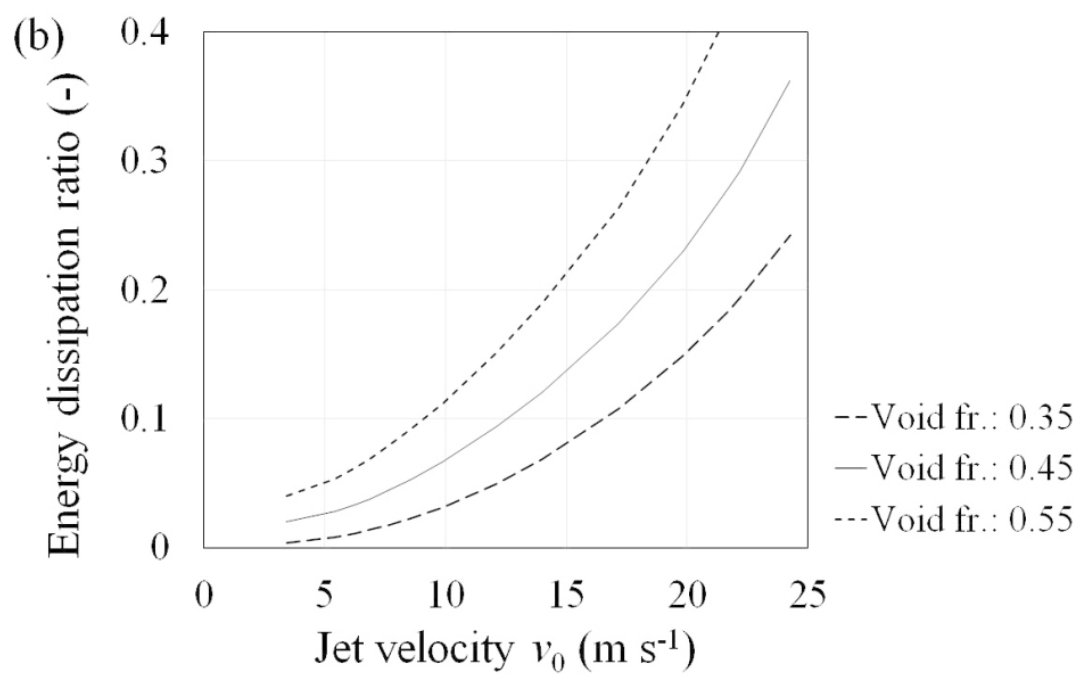

Figure 4 Energy dissipation ratio for isothermal and adiabatic conditions: (b) Adiabatic compression $117 \times 72 \mathrm{~mm}(300 \times 300$ DPI) 